\title{
Dual, orthogonal, backlit pinhole radiography in OMEGA experiments
}

\author{
C. C. Kuranz \\ University of Michigan, 2455 Hayward Street, Ann Arbor, Michigan 48109 \\ B. E. Blue \\ Lawrence Livermore National Laboratory, P.O. Box 808, Livermore, California 94550 \\ R. P. Drake \\ University of Michigan, 2455 Hayward Street, Ann Arbor, Michigan 48109 \\ H. F. Robey and J. F. Hansen \\ Lawrence Livermore National Laboratory, P.O. Box 808, Livermore, California 94550 \\ J. P. Knauer \\ University of Rochester, 250 East River Road, Rochester, New York 14623 \\ M. J. Grosskopf, C. Krauland, and D. C. Marion \\ University of Michigan, 2455 Hayward Street, Ann Arbor, Michigan 48109
}

(Received 9 May 2006; presented on 8 May 2006; accepted 3 August 2006; published online 26 October 2006)

\begin{abstract}
Backlit pinhole radiography used with ungated film as a detector creates x-ray radiographs with increased resolution and contrast. Current hydrodynamics experiments on the OMEGA Laser use a three-dimensional sinusoidal pattern as a seed perturbation for the study of instabilities. The structure of this perturbation makes it highly desirable to obtain two simultaneous orthogonal backlighting views. We accomplished this using two backlit pinholes each mounted $12 \mathrm{~mm}$ from the target. The pinholes, of varying size and shape, were centered on $5 \mathrm{~mm}$ square foils of $50 \mu \mathrm{m}$ thick Ta. The backlighting is by $K$-alpha emission from a $500 \mu \mathrm{m}$ square Ti or Sc foil mounted $500 \mu \mathrm{m}$ from the Ta on a plastic substrate. Four laser beams overfill the metal foil, so that the expanding plastic provides radial tamping of the expanding metal plasma. The resulting $\mathrm{x}$-rays pass through the target onto (ungated) direct exposure film (DEF). Interference between the two views is reduced by using a nose cone in front of the DEF, typically with a $9 \mathrm{~mm}$ Ta aperture and with magnets to deflect electrons. Comparison of varying types of pinholes and film exposures will be presented from recent experiments as well as an analysis of the background noise created using this experimental technique. () 2006 American Institute of Physics. [DOI: 10.1063/1.2351870]
\end{abstract}

\section{INTRODUCTION}

Backlit pinhole radiography ${ }^{1-5}$ is a technique in which laser irradiation produces $\mathrm{x}$-ray emission from an area of a target, after which a pinhole directs a small diverging beam of $\mathrm{x}$ rays through the object to be imaged and onto a detector. This technique, under development for a number of years, ${ }^{6-8}$ provides a higher intrinsic brightness than a traditional pinhole "box camera," permitting increased magnification and improved resolution. In principle, one can minimize the noise by detecting the $\mathrm{x}$ rays with film when a gated detector is not required. This requires, however, that the film be shielded at all times from all other sources of emission at energies above about $1 \mathrm{keV}$. In recent years, techniques to accomplish this have been developed. ${ }^{7}$

A natural extension of this technique is to obtain such data on the same experiment simultaneously along two orthogonal lines of sight. Such dual, orthogonal, backlit pinhole radiography is an essential tool in diagnosing threedimensional structure in hydrodynamics experiments. ${ }^{9}$ Our specific experiments consist of a three-dimensional (3D) sinusoidal perturbation having orthogonal rows of peaks and valleys. Obtaining two such images is significantly more difficult than backlit pinhole radiography in only one direction, because of cross-talk created by signals from the laserproduced plasmas that emit the diagnostic $\mathrm{x}$ rays. To our knowledge, our team is the first to have successfully obtained physics data simultaneously in two orthogonal directions by this technique. This present article discusses how this was accomplished.

\section{THE EXPERIMENTAL DESIGN}

The experiments each involved three targets. Two of these were backlit pinholes, described first. The third target was the hydrodynamic experiment, described next. A diagram of the pinhole structure can be seen in Fig. 1. The front surface consists of a $5 \mathrm{~mm}$ square foil of Ta with a pinhole in the center. The purpose of this large foil is to shield the diagnostic from emission from the hot plasma created by the laser beams that irradiate the rear surface of the structure. $500 \mu \mathrm{m}$ behind the pinhole is a $3 \mathrm{~mm}$ square $\mathrm{CH}$ foil attached to the Ta by plastic spacers on each side of the $\mathrm{CH}$. Attached to the rear surface of the plastic square is a $500 \mu \mathrm{m}$ 


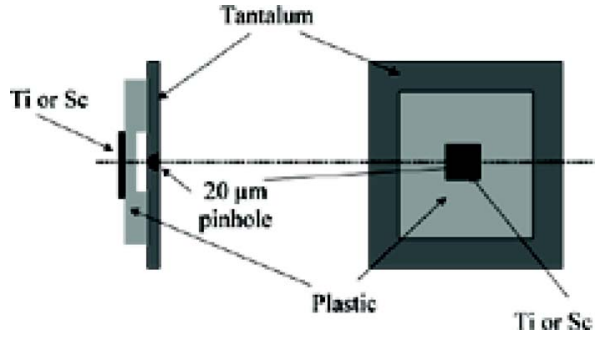

FIG. 1. (Color online) Design of backlit pinhole target from the side and rear views. The pinhole in the Ta is stepped with a large opening of $50 \mu \mathrm{m}$ and a smaller opening of $20 \mu \mathrm{m}$. $500 \mu \mathrm{m}$ behind the pinhole is a $50 \mu \mathrm{m}$ thick layer of plastic followed by a $500 \mu \mathrm{m}$ square foil of Ti or Sc.

square metal foil. Laser beams irradiate the foil and the surrounding plastic, creating metal plasma, plastic plasma, and the desired $\mathrm{Ti}$ or Sc $K$-alpha $\mathrm{x}$ rays of energy 4.51 or $4.09 \mathrm{keV}$, respectively.

The plastic foil serves three purposes. First, it provides plastic plasma that flows outward with the metal plasma ${ }^{10}$ and tamps the radial motion of this plasma. This has proven essential to avoid the exposure of the film by $\mathrm{x}$ rays from the metal plasma, seen around the edges of the Ta shield. Second, as the pressure from the laser-irradiated foil drives a shock through the plastic, this distributes the momentum and energy delivered to the metal foil over a larger mass of material, reducing the tendency to create shrapnel when this material strikes the Ta. Third, the plastic can be expected to absorb most of the energetic electrons produced by laserplasma interactions in the metal plasma. Even so, experience has shown that the irradiance of the metal foil cannot exceed a few times $10^{14} \mathrm{~W} / \mathrm{cm}^{2}$ without creating greatly increased noise. This particular experiment uses both $\mathrm{Ti}$ and $\mathrm{Sc}$ foils because the opacity of the target structures of interest is different along the two lines of sight.

The pinhole in the Ta serves as a filter, only allowing $\mathrm{x}$-rays pointing directly at the target to pass through. These specific experiments used a pinhole aperture of $10 \mu \mathrm{m}$ or a stepped pinhole with the large side being $50 \mu \mathrm{m}$ and the smaller opening being $20 \mu \mathrm{m}$. The step refers to a large hole on one side of the Ta and a smaller hole on the other. Using a stepped structure (or a tapered pinhole), as opposed to a straight pinhole, produces $\mathrm{x}$ rays having a larger cone angle and a more uniform intensity. This reduces the sensitivity of the pinhole backlighters to rotational alignment (though their alignment remains quite demanding). By comparison, the $10 \mu \mathrm{m}$ straight pinhole allows for better resolution than the $20 \mu \mathrm{m}$ stepped pinhole achieves, but any tilt in the foil will quickly decrease the source size, limiting the number of photons on the detector. A comparison of intensities from these two pinhole types is shown later in this article.

Accomplishing backlit pinhole radiography along two orthogonal lines of sight proved significantly more demanding than using only one line of sight. Using two backlighters is very difficult because any illumination from one will interfere with the other. This cross-talk effect is further complicated when using ungated film. In previous attempts, gated diagnostics were used and fired at different times during the experiment in an effort to prevent interference. However, in some cases, it has been noted that standard

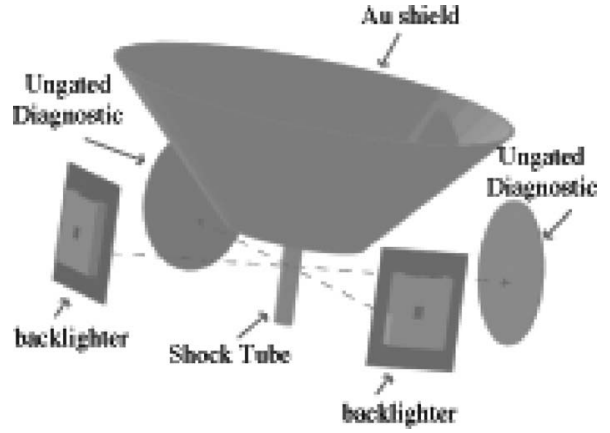

FIG. 2. Positions of targets and diagnostics of experiment. The large, center target is the hydrodynamics target with the two backlighters perpendicular to the target and the other backlighter. There is a Static Pinhole Camera Array (SPCA) loaded with DEF on the opposite side of the target from each of the backlighters. The large gold cone on the main target shields the ungated diagnostics from the laser beams and hot plasma that is created during the experiment.

microchannel-plate framing cameras provided insufficient extinction of the ungated signals and so this approach generally failed. In order to reduce the cross-talk, the present experiments protected each film holder with a nose cone. The narrow front end of the nose cone was located 1/3 of the $(228 \mathrm{~mm})$ distance from the target to the film. At this front end a Ta shield having a $9 \mathrm{~mm}$ circular aperture was mounted, and magnetic material was included to deflect any electrons away from the film. With the two pinholes each displaced $12 \mathrm{~mm}$ from the target axis along orthogonal lines of sight, the $9 \mathrm{~mm}$ aperture was just sufficient to prevent the exposure of a portion of a given film pack by emission from the orthogonal backlighter. There was apparently some remaining exposure, presumably due to scattering of the $\mathrm{x}$ rays, but this was small. With these techniques, it is possible to obtain data simultaneously along both lines of sight.

Figure 2 shows the configuration of the experiment using this technique. The hydrodynamics target is placed so that the laser beams that deliver energy to it are focused at the center of the OMEGA chamber. The two backlit pinhole structures are placed $12 \mathrm{~mm}$ from the target and $90^{\circ}$ from each other. The placement of the film allows for a magnification of about 20 on the radiograph. During the experiment, ten OMEGA laser beams irradiate the target, and the backlighters are irradiated after a delay of 7-40 ns. The group of beams irradiating the rear side of each pinhole structure consists of two to four OMEGA laser beams with a $1 \mathrm{~ns}$ pulse, 200-400 J/beam, and 1000-1200 $\mu \mathrm{m}$ spot size. The diagnostic $\mathrm{x}$ rays pass through the target and onto direct exposure film (DEF). DEF is well characterized; by using the model of Henke et al. the optical density of the film can be converted to intensity in photons $/ \mu \mathrm{m}^{2}{ }^{11}$ In the film holder up to three pieces of film are layered on top of one another and behind $\mathrm{Be}$, plastic, and Ti or Sc light shields. The film is $\sim 50 \mathrm{~mm}$ in diameter and allows a field of view of about $1500 \mu \mathrm{m}$ of the target.

\section{RESULTS AND DISCUSSION}

Figure 3 shows radiographs from the experiment. These images, taken at $17 \mathrm{~ns}$ after the drive beams fired, are from 


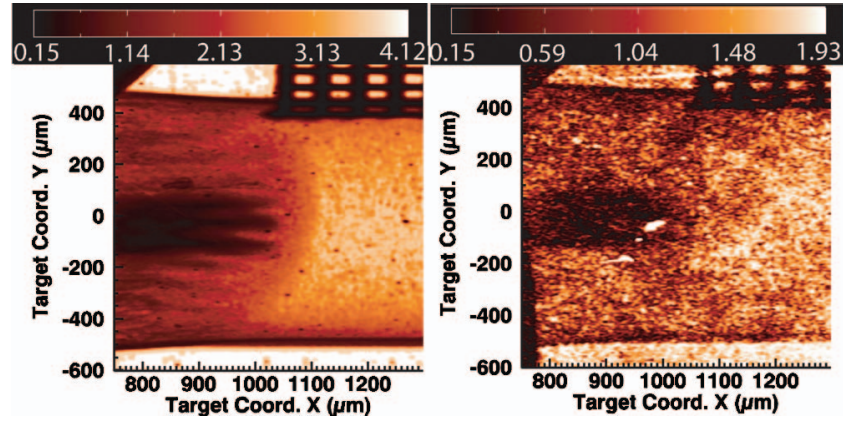

FIG. 3. (Color) Radiographic images from the experiment taken at $17 \mathrm{~ns}$ after the drive beams have fired. These radiographs are from the same experiment with (a) being the top layer of DEF and (b) being directly behind it. Each image has a color bar associated with it that is in units of photons/ $\mu \mathrm{m}^{2}$.

the same laser shot. Figure 3(a) is the first layer of film closest to the experiment itself and Fig. 3(b) is the layer that was directly behind it. Each image has a horizontal color bar showing the intensity in units of photons $/ \mu \mathrm{m}^{2}$. In both images the dark area in the center of the image is the leastexposed region of interest, created by a long, rectangular, "tracer strip" of brominated plastic that is a comparatively strong absorber of x rays. In Fig. 3(a) the spikes, long dense fingers moving to the right, and bubbles, less dense structures moving to the left, from the Rayleigh-Taylor instability are more clearly seen. The shock front is also seen at $\sim 950 \mu \mathrm{m}$.

We evaluated the exposure and noise in the calibrated radiographic images as follows. Line outs were taken across 1 pixel of the radiograph from a uniformly exposed area on the film. In this case, 1 pixel is equal to $22 \mu \mathrm{m}$ on the film and the experiment had a magnification of 20, therefore, 1 pixel is $1.1 \mu \mathrm{m}$ on the target. The mean and standard deviation were calculated from this line out. The same process was done on an area where there should be no signal, in this case, behind the gold shield. The mean exposure was considered to arise from either background noise or processing of the film. The number of photons $/ \mu \mathrm{m}^{2}$ of interest was found by subtracting the intensity from noise signal from the intensity across a uniformly exposed area. From Fig. 3(a) the average number of photons $/ \mu \mathrm{m}^{2}$ was 2.99 vs 0.03 in Fig. 3(b). Clearly, the first layer of film acts as a filter and fewer photons get to the second layer of film. This information is shown in Table I along with anticipated and observed values of photon statistics. These values are also listed for several

TABLE I. Estimated and observed values of the Poisson noise, $1 / \sqrt{ }(N)$ for four different experiments. Values for rows 1 and 2 are from two layers of DEF from the same experiment. Rows 3 and 4 were similar experiments except for difference pinhole apertures.

\begin{tabular}{cccc}
\hline \hline Description & Mean & $\begin{array}{c}\text { Expected } \\
1 / \sqrt{ }(N)\end{array}$ & $\begin{array}{c}\text { Obs. } \\
1 / \sqrt{ }(N)\end{array}$ \\
\hline First layer of DEF film & 2.990 & 0.026 & 0.055 \\
Second layer of DEF film & 0.033 & 0.250 & 0.212 \\
$20 \mu \mathrm{m}$ stepped pinhole & 1.082 & 0.044 & 0.029 \\
$10 \mu \mathrm{m}$ straight pinhole & 0.174 & 0.109 & 0.070 \\
\hline \hline
\end{tabular}

other experiments, including a comparison between a pinhole with an aperture of $50 \mu \mathrm{m}$ stepped to $20 \mu \mathrm{m}$ and a $10 \mu \mathrm{m}$ straight pinhole.

It is also of interest to estimate the expected number of photons/pixel. Using conclusions made by Kyrala et al. the conversion efficiency of Ti is $5.66 \times 10^{11}$ photons $/ \mathrm{J}$ sphere. $^{12}$ The laser beams striking the $\mathrm{Ti}$ foil have a total energy of $800 \mathrm{~J}$ and the area of the foil is $2.5 \times 10^{5} \mu \mathrm{m}^{2}$. If we estimate that only $\sim 50 \%$ of these photons get transmitted through the foil and then pass through the pinhole with an area of $100 \pi \mu \mathrm{m}^{2}$. The distance to the film is $\sim 230 \mathrm{~mm}$ and 1 pixel is equal to $484 \mu \mathrm{m}^{2}$ on the film, so there are 9.15 $\times 10^{-9}$ srs/pixel. This gives about 200 photons/pixel or about 0.5 photons $/ \mu \mathrm{m}^{2}$, which does not take into account the plastic, Be, or Ti light filters. This is in good agreement with the values of Table I.

To calculate the values in Table I let $\mathrm{N}$ be the number of photons/pixel. We find $\mathrm{N}$ by multiplying the average number of photons $/ \mu \mathrm{m}^{2}$ by the area in 1 pixel, $484 \mu \mathrm{m}^{2}$. If Poisson noise were the only type of noise in this radiograph then the standard deviation divided by the signal would be $1 / \sqrt{ }(N)$. Table I shows this value, and shows for comparison the observed noise obtained by dividing the standard deviation of the values obtained in the line out described above by the mean, background-subtracted signal. The comparison of the expected and observed values is important because it determines whether the majority of noise on the radiograph is from Poisson noise or if there is a larger noise source in the experiment.

The anticipated and observed values agree within a factor of 2 and very closely agree in some cases. It should be noted that the film used in these experiments had been stored over a long period and this increases the fog level of the film. As the fog level increases the film has a lower dynamic range. There is also an area of uncertainty in the difference among the film processing techniques used by Henke et al. and the Laboratory for Laser Energetics. Other possibilities as to the difference between the theoretical and observed Poisson noise include saturation of the film if the actual Poisson noise is lower than the theoretical value. The first layer of film in Fig. 3(a) is presumably close to this point, although the observed noise is reasonably consistent with the expected Poisson noise. In this image, a line out of an area outside the target is flat meaning the film is fully exposed. In this experiment the lasers striking the metal foil had an intensity of $6.4 \times 10^{14} \mathrm{~W} / \mathrm{cm}^{2}$ Compare this to the last line in the table, which is the case of the $20 \mu \mathrm{m}$ stepped pinhole that had a lower energy/beam as well as a larger spot size to decrease the intensity to $2.2 \times 10^{14} \mathrm{~W} / \mathrm{cm}^{2}$. The experiment using a $10 \mu \mathrm{m}$ straight pinhole also had an intensity of 2.2 $\times 10^{14} \mathrm{~W} / \mathrm{cm}^{2}$; however, the area of the pinhole was decreased by a factor of 4 making the radiograph dim. In all cases in Table I the observed noise is within a factor of 2 of the Poisson noise, meaning that other sources of noise in the experiment, if present, are not dominant.

\section{SUMMARY}

This article has discussed the design and geometry needed for dual, orthogonal backlit radiography. Results 
from these experiments show that only $<1 \%$ of the backlighter beam energy exposes the film and the Poisson noise is the dominant source of noise. In future experiments, the backlighter structure will have a Ta foil with $10 \mu \mathrm{m}$ tapered pinholes. The taper is similar to the step, but has a smoother transition. These pinholes will have a large opening of $\sim 20 \mu \mathrm{m}$ tapered to $10 \mu \mathrm{m}$.

\section{ACKNOWLEDGMENTS}

The authors acknowledge useful interactions with and the significant technical contributions to backlit pinhole radiography of J. M. Foster, T. S. Perry, C. Sorce, and S. Sublett. The authors would also like to thank the technical staff at the OMEGA Laser Facility. This work is supported by the National Nuclear Security Agency under DOE Grant No. DE-FG03-99DP00284 and DE-FG03-00SF22021, and by other grants and contracts.
${ }^{1}$ C. L. S. Lewis and J. McGLinchey, J. Opt. Commun. 53, 179 (1985).

${ }^{2}$ D. Oneill, C. L. S. Lewis, D. Neely, S. J. Davidson, S. J. Rose, and R. W. Lee, Phys. Rev. A 44, 2641 (1991).

${ }^{3}$ K. S. Budil et al., Rev. Sci. Instrum. 67, 485 (1996).

${ }^{4}$ J. Workman et al., Rev. Sci. Instrum. 75, 3915 (2004).

${ }^{5}$ B. E. Blue, J. F. Hansen, M. T. Tobin, D. C. Eder, and H. F. Robey, Rev. Sci. Instrum. 75, 4775 (2004); J. Fincke, J. R. Workman, G. A. Kyrala, and T. Pierce, Appl. Opt. 44, 859 (2005).

${ }^{6}$ A. B. Reighard, R. P. Drake, K. K. Dannenberg, J. P. Knauer, S. Bouquet, and L. Boireau, Phys. Plasmas 12, 056313 (2005).

${ }^{7}$ J. M. Foster et al., Astrophys. J. Lett. 634, L77 (2005).

${ }^{8}$ B. E. Blue et al., Phys. Plasmas 12, 056317 (2005).

${ }^{9}$ R. P. Drake et al., Phys. Plasmas 11, 2829 (2004).

${ }^{10}$ M. J. Herbst, P. G. Burkhalter, J. Grun, R. R. Whitlock, and M. Fink, Rev. Sci. Instrum. 53, 1418 (1982).

${ }^{11}$ B. L. Henke, J. Y. Uejio, G. F. Stone, C. H. Dittmore, and F. G. Fujiwara, J. Opt. Soc. Am. B 3, 1540 (1986).

${ }^{12}$ G. A. Kyrala, K. Klare, and J. Workman, Rev. Sci. Instrum. 74, 2182 (2003). 